\title{
Intrarectal Xyloglucan Administration Reduces Disease Severity in the Dextran Sodium Sulfate Model of Mouse Colitis
}

\author{
Edward A Ross $\mathbb{1}^{1}$ \\ Madelyn H Miller (D) ${ }^{2}$ \\ Allison Pacheco ${ }^{2}$ \\ Alicia R Willenberg (iD) \\ Justine T Tigno-Aranjuez (D) ${ }^{2}$ \\ Kaitlyn E Crawford (iD ${ }^{3}$ \\ 'Department of Internal Medicine, \\ College of Medicine, University of \\ Central Florida, Orlando, FL, USA; \\ ${ }^{2}$ Immunity and Pathogenesis Division, \\ Burnett School of Biomedical Sciences, \\ University of Central Florida, Orlando, \\ FL, USA; ${ }^{3}$ Department of Materials \\ Science and Engineering, College of \\ Engineering and Computer Sciences, \\ University of Central Florida, Orlando, \\ FL, USA
}

Background: The pathophysiology of inflammatory bowel diseases remains poorly understood and treatment remains suboptimal for many patients. We hypothesize that the inflammatory milieu secondarily prolongs the injury and attenuates healing. We propose primary or adjuvant therapy with biocompatible adhesives to restore a barrier to protect submucosal structures, particularly stem cells.

Methods: We used the well-described mouse dextran sodium sulfate (DSS) model of colitis resembling human ulcerative colitis to test the therapeutic efficacy of intrarectal administration of the tamarind plant-derived xyloglucan (TXG) polymer adhesive which underwent extensive analytic characterization. Mice in control, DSS-only, TXG-only, and DSS + TXG groups were studied for gross (weight, blood in stool, length of colon) and multiple histologic parameters.

Results: Compared to DSS-only mice, TXG prevented the weight loss, occurrence of blood in the stool and colon shortening, with all those parameters not being statistically different from treatment naïve animals. Histologically, there was dramatic and highly statistically significant reduction in the total inflammatory index and protection from goblet cell loss, cellular infiltration, crypt abscess formation, epithelial erosion, granulation tissue, epithelial hyperplasia crypt irregularity and crypt loss. The TXG purity and characterization were established by nuclear magnetic resonance, infrared spectroscopy, differential scanning calorimetry, and texture analysis.

Conclusion: The striking attenuation of disease severity by intrarectal TXG use warrants future investigations of natural bioadhesives with well-established high safety profiles, and which could potentially be derivatized to include therapeutically active moieties for local drug delivery.

Keywords: ulcerative colitis, dextran sodium sulfate, DSS, xyloglucan, tamarind, inflammatory bowel disease

\section{Plain Language Summary}

In a mouse model resembling ulcerative colitis, intrarectal tamarind xyloglucan demonstrated profound protection for multiple whole animal and histologic parameters. These findings support the use of this plant-based polymer bioadhesive mucosal barrier as primary or adjuvant therapy for human disease.

\section{Introduction}

Department of Medicine, College of Medicine, University of Central Florida, 6850 Lake Nona Blvd, Orlando, FL, 32827, USA

Email Edward.ross@ucf.edu
Despite advances in understanding the pathophysiology of inflammatory bowel diseases (IBD) and the development of new pharmaceuticals that target immunologic mechanisms, treatment remains suboptimal for many patients and do not 
specifically address protection of the colonic epithelial barrier We hypothesize that even with medications effectively targeting the underlying (eg, autoimmune and inflammatory) processes, healing is attenuated by the diseased milieu: ${ }^{1}$ denudation of the epithelium; loss of the cellular and mucin barrier; infiltration by bacteria, toxins, cytokines and leukocytes; then submucosal inflammation and necrosis. We believe that the loss of stem cells in the crypts has a key role in thwarting recovery, as that process normally depends on precursor differentiation and proliferation. The goal of this study is to use a biocompatible adhesive as a protective barrier to chemical and cellular inflammatory compounds in the colon so as to shield submucosal structures, particularly stem cells. Rather than using inert coatings, a future approach is to administer bioadhesives that have innate anti-inflammatory activity or can be derivatized to deliver topical targeted pharmacologic treatment. The latter is an attractive goal since in situ medicinals may avoid adverse effects from systemic administration.

Bioadhesives have a long history of medicinal use, and many are derived from plants rather than being prescription-based synthetic pharmacologic agents. Some natural compounds have been used as mechanical barrier coatings (for disorders of the eye, skin and stomach) and others are purported to have anti-inflammatory effects. ${ }^{2-4}$ Many plant-derived moieties have a polymeric glucose backbone with side chains that determine tissue interactions. Their similarities to human colon-protective mucins make these starches attractive candidates for intestinal adhesive coatings, and we chose to study xyloglucan (XG, generically) extracted from tamarind seed (TXG, specifically). ${ }^{5,6}$ We hypothesized that XG would have therapeutic benefit to mice having undergone mucosal injury in an experimental model of IBD. An affirmation of this approach is that tamarind plants have centuries of use as natural remedies for a variety of gastrointestinal disorders. We administered TXG to mice with the dextran sulfate-induced model of colitis that resembles human ulcerative colitis (UC) in order to determine both in vivo effects as well as the impact on tissue morphology.

\section{Materials and Methods \\ Mice}

C57BL/6J mice were obtained from Jackson Laboratories (Bar Harbor, Maine, USA) and were bred and housed in the pathogen-free, AALAC-accredited, animal facility at the University of Central Florida (UCF) Health Science Campus at Lake Nona, FL. All animal procedures were performed in accordance with the guidelines of the UCF Institutional Animal Care and Use Committee using an approved protocol (PROTO202000006). Mice were females 8 weeks old at the start of the experiment. They were weighed daily for the duration of the treatment to monitor weight loss. Fecal samples were collected to assess blood in the stool using Hemoccult II slides (Beckman Coulter). Blood presence was assessed based on a scale of $0-2$ ( 0 being no blood, 2 being severe).

\section{DSS and TXG Administration}

The dextran sodium sulfate (DSS) was obtained as a powder from TdB Consultancy AB (Uppsala, Sweden), with their analysis reporting an average molecular weight of 47,730 Daltons (range 35,000-50,000) with $18 \%$ sulfur substitution. To assure adequate delivery (directly) to the colon and to avoid potential confounding factors from oral administration (eg, interactions with DSS, hepatobiliary or intestinal enzymes) the TXG was given intrarectally with the animals positioned horizontally for approximately 3-5 minutes. Mice were split into the following groups $(n=13$ each): no treatment (naive), intrarectal administration of TXG only, treatment with DSS only, or intrarectal administration of TXG and treatment with (DSS+TXG). On D0, DSS-only and DSS+TXG mice were started on $2.0 \%$ DSS in water and other water sources were removed. Water bottles were weighed to ensure mice were consuming the water. Mice were thus continued on DSS in water until they were returned to normal water at the end of D6. TXGonly and DSS + TXG mice were administered $\sim 100 \mu \mathrm{L} 2 \%$ TXG, as a therapeutic, intrarectally on D2 and D5 of the experiment. Mice were euthanized on D12 for tissue harvesting.

\section{TXG Preparation and Analysis}

Based on limitations of some prior published literature using incompletely characterized plant derivatives, it was important to analyze the reagents in this study for characterization of the TXG and potential impurities. The TXG was sourced as the tamarind-derived powder (TCI America, Portland, OR) and used as received. Two percent TXG solutions were prepared by stirring at $65^{\circ} \mathrm{C}$ in deionized water overnight. All TXG materials were autoclaved prior to use. Nuclear Magnetic Resonance (NMR): ${ }^{1} \mathrm{H}$ NMR spectroscopy was carried out at room temperature with a Bruker 
AVIII-400 MHz spectrometer. Samples were prepared at $2 \mathrm{mg} / \mathrm{mL}$ in $\mathrm{D}_{2} \mathrm{O}$. Attenuated Total Reflectance Fourier Transform Infrared spectroscopy (ATR-FTIR): Measurements were carried out using an Agilent Cary 630 spectrometer fitted with a diamond ATR crystal. Spectra are based on 128 scans with $8 \mathrm{~cm}^{-1}$ resolution. Differential Scanning Calorimetry (DSC): Measurements were carried out using a Netzsch Polyma 214 system. Thermal properties of TXG were tested in sealed hermetic pans with an average material weight of $6 \mathrm{mg}$, each run alongside an empty pan for reference. The temperature program employed was as follows: two heating and two cooling cycles, each with a temperature ramp of $20{ }^{\circ} \mathrm{C} / \mathrm{min}$ with dry nitrogen gas and temperature range of $0{ }^{\circ} \mathrm{C}$ to $330^{\circ} \mathrm{C}$. The second heat and first cool cycles are reported. Texture Analysis: Measurements were carried out using a Texture Technologies, TA XT-plus texture analyzer. The sample probe was a stainless-steel Peltier plate with $6.35 \mathrm{~mm}$ diameter surface area. The probe was set to $10 \mathrm{~mm} / \mathrm{min}$ and values were reported as an average of 3 runs.

\section{Histology and Scoring}

At the end of the model, mice were euthanized and colons were harvested. Colon lengths were measured and gently flushed using a transfer pipet with PBS. Colons were cut lengthwise, opened and fixed flat for 20 mins in $10 \%$ buffered formalin and subsequently rolled in swiss-roll fashion (Figure 1), placed into histocassettes and further fixed in 10\% buffered formalin overnight. Colons $(\mathrm{n}=10$, each group) were sent to AML labs (St. Augustine, FL) for paraffin embedding, microtome sectioning $(5 \mu \mathrm{M})$, and hematoxylin and eosin (H\&E) staining. To assess inflammation, H\&E sections of the distal colon $(n=10$ from each experimental group) were scored blindly based on a modified histopathological scoring system. ${ }^{7}$ Since intrarectal routes of medication administration are known to have better delivery to the left colon, ${ }^{8}$ scoring was performed separately for the left, right and total colon. The following parameters were added together for a total inflammatory score $0-43(43=$ most severe): severity of inflammatory infiltrate and extent of inflammatory cell infiltrate $(0-5)$, epithelial cell hyperplasia $(0-4)$, goblet cell loss $(0-3)$, cryptitis $(0-3)$, crypt abscess $(0-$ $5)$, erosion ( $0-3)$, ulceration $(0-5)$, granulation tissue $(0-5)$, irregular crypts $(0-5)$, crypt loss $(0-5)$.

\section{Results \\ TXG Characterization}

To characterize and validate the purity of the TXG obtained for these studies, rigorous analysis was performed. The ${ }^{1} \mathrm{H}$ NMR spectrum exhibits peaks between $3.1 \mathrm{ppm}$ and 4.1 $\mathrm{ppm}$, and at ca. $4.6 \mathrm{ppm}$. This peak range is characteristic of polysaccharides and consistent with the literature for the sugars in TXG (ie, galactose, glucose, and xylose) ${ }^{5,9}$ (Figure 2A). ATR-FTIR spectra shows a broad peak at $3351 \mathrm{~cm}^{-1}$ indicative of an $\mathrm{O}-\mathrm{H}$ stretch, followed by $\mathrm{C}-\mathrm{H}$, $\mathrm{C}-\mathrm{O}$, and $\mathrm{C}-\mathrm{C}$ vibrations at $2889 \mathrm{~cm}^{-1}(\mathrm{CH}), 1643 \mathrm{~cm}^{-1}$ and $1373 \mathrm{~cm}^{-1}$, and $1034 \mathrm{~cm}^{-1}$, respectively (Figure 2B). DSC curves (second heat and first cool) do not show glass transition or melting temperatures in the range between $25^{\circ} \mathrm{C}$ and $330^{\circ} \mathrm{C}$. In air and under nitrogen the TXG samples begin to yellow above ca. $150^{\circ} \mathrm{C}$, and become increasingly brown until blackening above $330^{\circ} \mathrm{C}$. The endothermic peak at 96.7 ${ }^{\circ} \mathrm{C}$ is consistent with the anticipated water content. A second endothermic peak occurs at $168.7^{\circ} \mathrm{C}$. Overlapping exothermic peaks occur at $330{ }^{\circ} \mathrm{C}$. Here, peaks are only observed during the first heating cycle. No peaks are present in the cooling cycles (not shown) or subsequent heating cycles (Figure 2C). Repeated DSC characterization of dried TXG was carried out at lower temperatures down to $160{ }^{\circ} \mathrm{C}$ (not shown). In such cases, no peaks were observed during the second heating, and the endothermic peak near $100{ }^{\circ} \mathrm{C}$ was no longer present. Texture analysis of $2 \%$ TXG solutions $(\mathrm{n}=3)$ exhibit tack of $61.1 \pm 0.9 \mathrm{~g}$, adhesiveness of $4.3 \pm 0.1$, and work of adhesion of $65.9 \pm 1 \mathrm{~g}$-s.

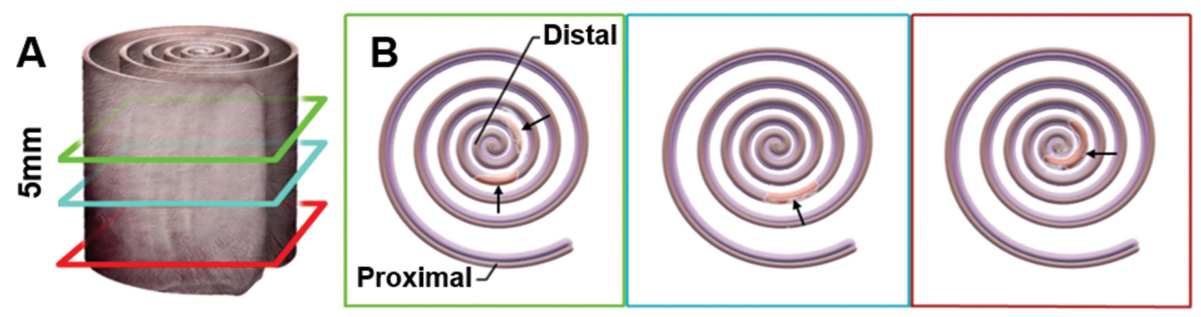

Figure I Methodology for tissue preparation and histology. Swiss Roll: (A) The entire colon is cut down the length, spread out and wrapped starting with the distal end (rectum) and rolled ending with the most proximal end on the outside. (B) The tissue was processed through paraffin, sectioned at $5 \mu \mathrm{M}$ and stained with $\mathrm{H} \& \mathrm{E}$; arrows indicate areas of focal damage in the DSS model. 
A

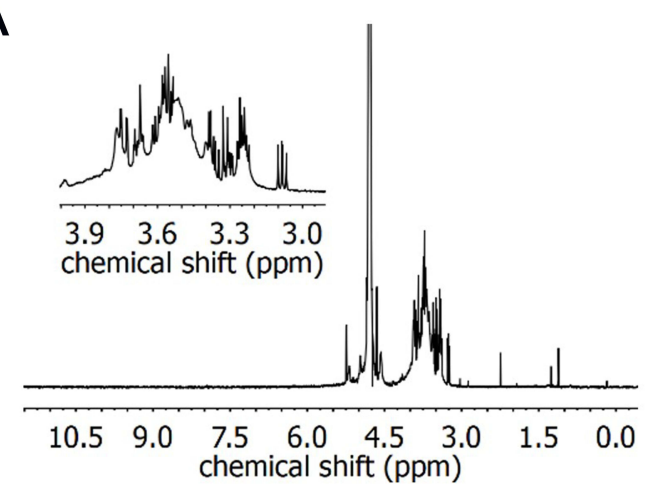

B

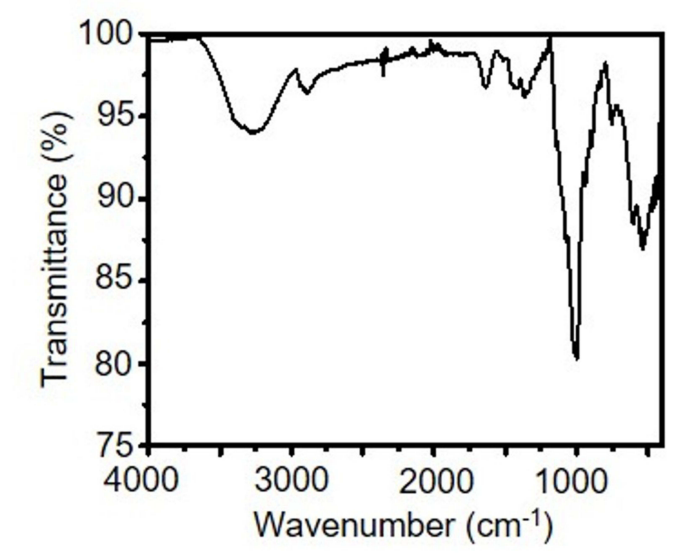

C

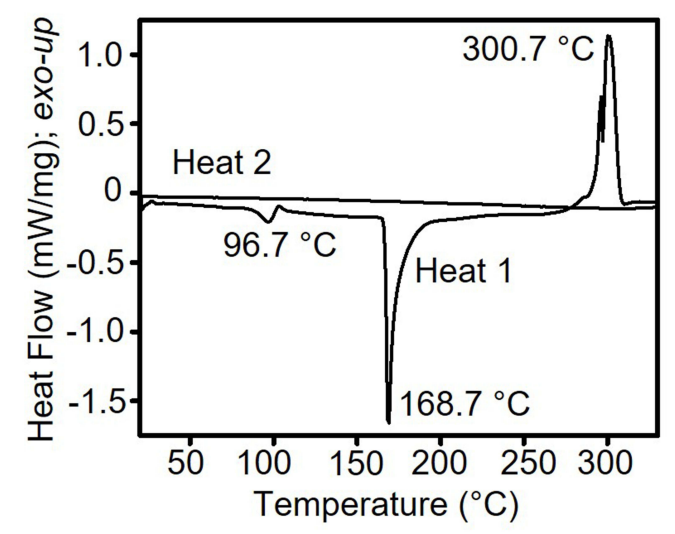

Figure 2 TXG Material Characterization. (A) ' $\mathrm{H}$ NMR of TXG at $400 \mathrm{MHz}$ in $\mathrm{D}_{2}$ O. (B) ATR-IR of TXG. (C) DSC of TXG at $20^{\circ} \mathrm{C} / \mathrm{min}$.

\section{In vivo Studies}

To test for the benefits of TXG the DSS mouse model incorporated appropriate controls and we studied clinical parameters as well as these extensive histologic analyses.

\section{Body Weight (Figure 3A)}

Animals treated only with the TXG polymer had wholebody weights unchanged from the control naïve animals
$19.2 \pm 1.0 \mathrm{~g}$. After DSS administration, mice demonstrated a loss in body weight typically reported in this colitis model of approximately $2 \mathrm{~g}$ (weight: $17.5 \pm 0.8 \mathrm{~g}$, p 0.018) that was greatest at day 10. The DSS + TXG group had weights similar to the naïve animals (19.4 \pm $1.3 \mathrm{~g}, \mathrm{p}>0.8$ ). Blood in stool (Figure 3B): Naïve and TXG-only animals had no detectable blood in their stool using the semi-quantitative hemoccult testing (score 0). The DSS-treated animals had bleeding with a mean score of $1.0 \pm 0.91$ with $\mathrm{p}<0.0001$ compared to the naïve animals which had no detectable bleeding. The DSS +TXG-treated mice were protected, being no different than the naïve mice $(p>0.9)$. Colon length (Figure 3C): On post-mortem, the length of the colon of the TXG-only mice was no different from that of naïve animals $(8.8 \pm$ $0.5 \mathrm{~cm})$. The DSS group had significantly shortened bowel at $7.6 \pm 0.4 \mathrm{~cm}, \mathrm{p}<0.0001$. The bowel length of the DSS + TXG mice was statistically longer $(8.7 \pm 0.8 \mathrm{~cm}, \mathrm{p}=$
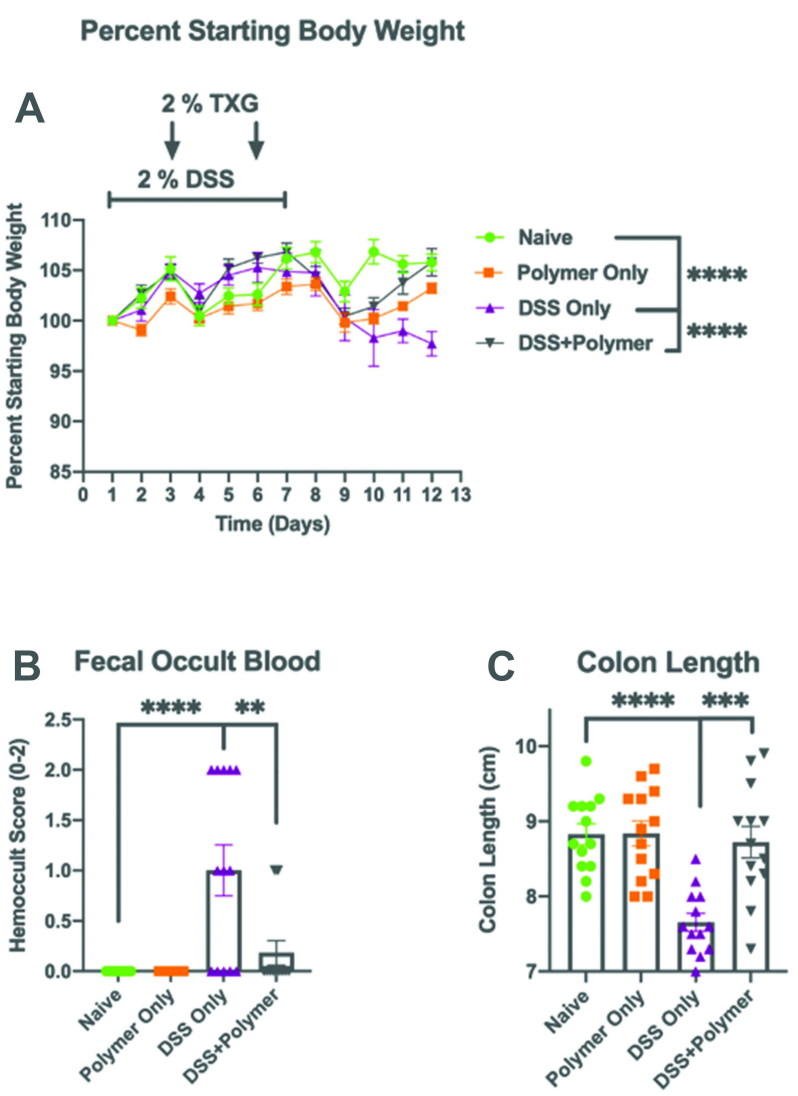

Figure 3 Intrarectal administration of TXG improves disease severity in a mouse model of colitis. C57BL/6J mice were left untreated or provided $2 \%$ DSS via drinking water for 7 days to induce colitis. On days 2 and 5, 2\% TXG was administered intrarectally. (A) Intrarectal TXG polymer administration significantly reverses DSS-induced weight loss on day I2. (B) Intrarectal TXG polymer administration significantly reduces incidence of fecal occult blood. (C) TXG polymer administration prevented DSS-induced colon shortening. $\mathrm{N}=13$ mice/group. Data were analyzed using One-way ANOVA with ${ }^{*} \mathrm{p}<0.01$, ${ }^{*} \mathrm{p}<0.001$, $* * * \mathrm{p}<0.000$ I. 
0.0002) than that of the DSS-only animals, but not statistically different from the naïve animals $(p>0.97)$. These results indicate that TGX was beneficial in reducing DSSinduced colitis health-related effects when given therapeutically on D2 and D5 of the model.

\section{Histologic Studies (Figure 4A-K Showing Scores from Representative Micrographs in Figure 5A-D)}

Exposure to TXG alone had no discernible effect on any component of the histology compared to control animals; all $\mathrm{p}>0.05$ when compared to the control group. Notably, when the histologic analyses were stratified into right versus left colonic inflammation, it was observed that the majority of the inflammation (as well as the beneficial effects of TXG) demonstrated in scores for the total colon were in fact attributable to the left colon, and those data are described below.

\section{Inflammation}

The total inflammatory (TI) index for the total colon showed severe involvement with DSS alone (24.9 \pm 6.9$)$ compared to $1.5 \pm 2.5$ in naïve mice and was remarkably

\section{A Total Inflammatory Index}
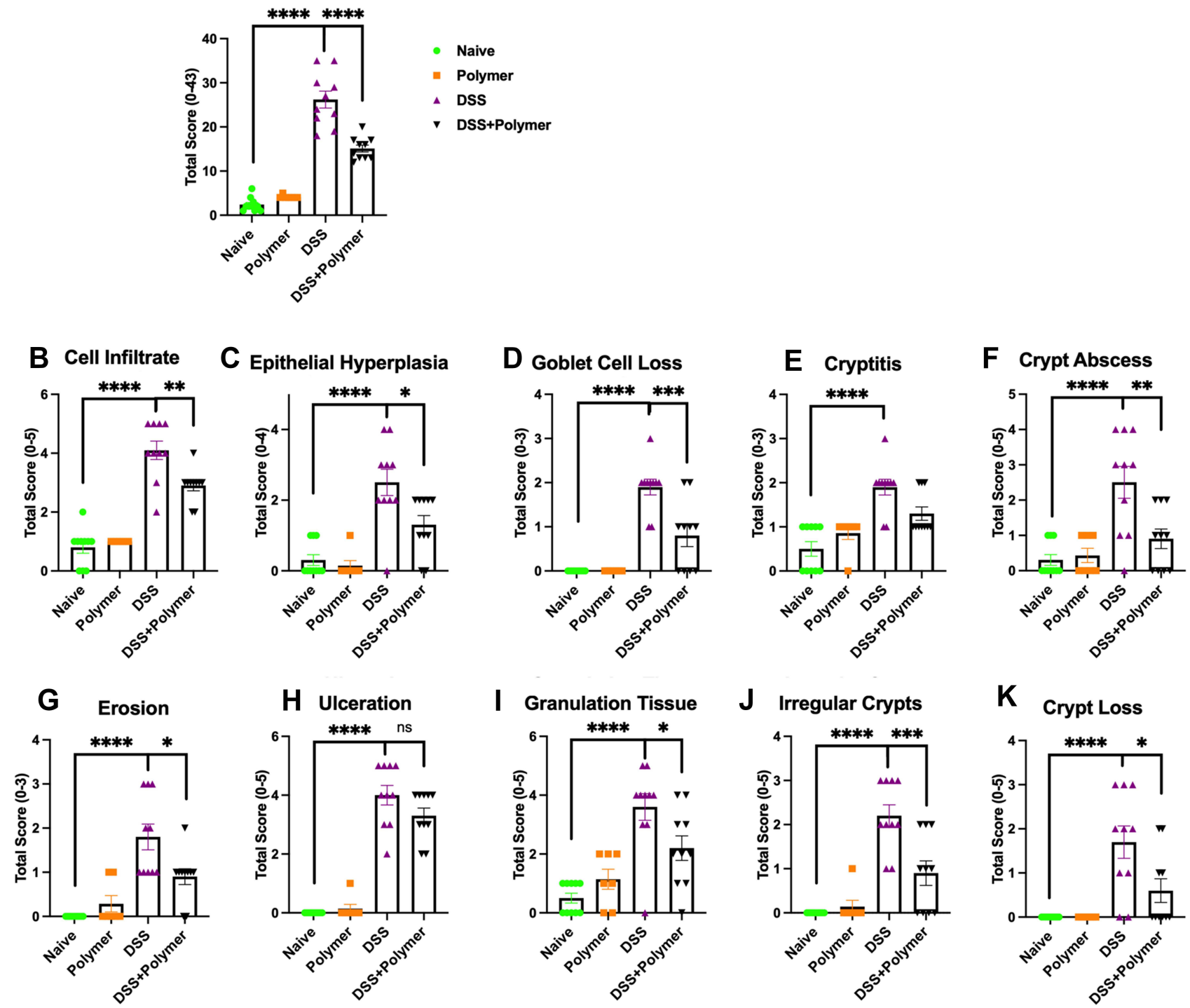

Figure 4 Intrarectal administration of TXG improves multiple histopathological parameters. From scoring of the left colon, (A) Total inflammatory index was aggregated from multiple pathological features including (B) Cell infiltration, (C) Epithelial hyperplasia, (D) Goblet Cell Loss, (E) Cryptitis, (F) Crypt Abscess, (G) Erosion, (H) Ulceration, (I) Granulation Tissue, (J) Irregular Crypts and (K) Crypt Loss. Slides were scored by a trained observer blinded to the sample treatment. The range of possible scores are indicated for each feature. $N=13$ mice/group. Data were analyzed using One-way ANOVA with $*_{p}<0.05, * * p<0.01, * * * p<0.001, *^{* * *} p<0.0001$. 

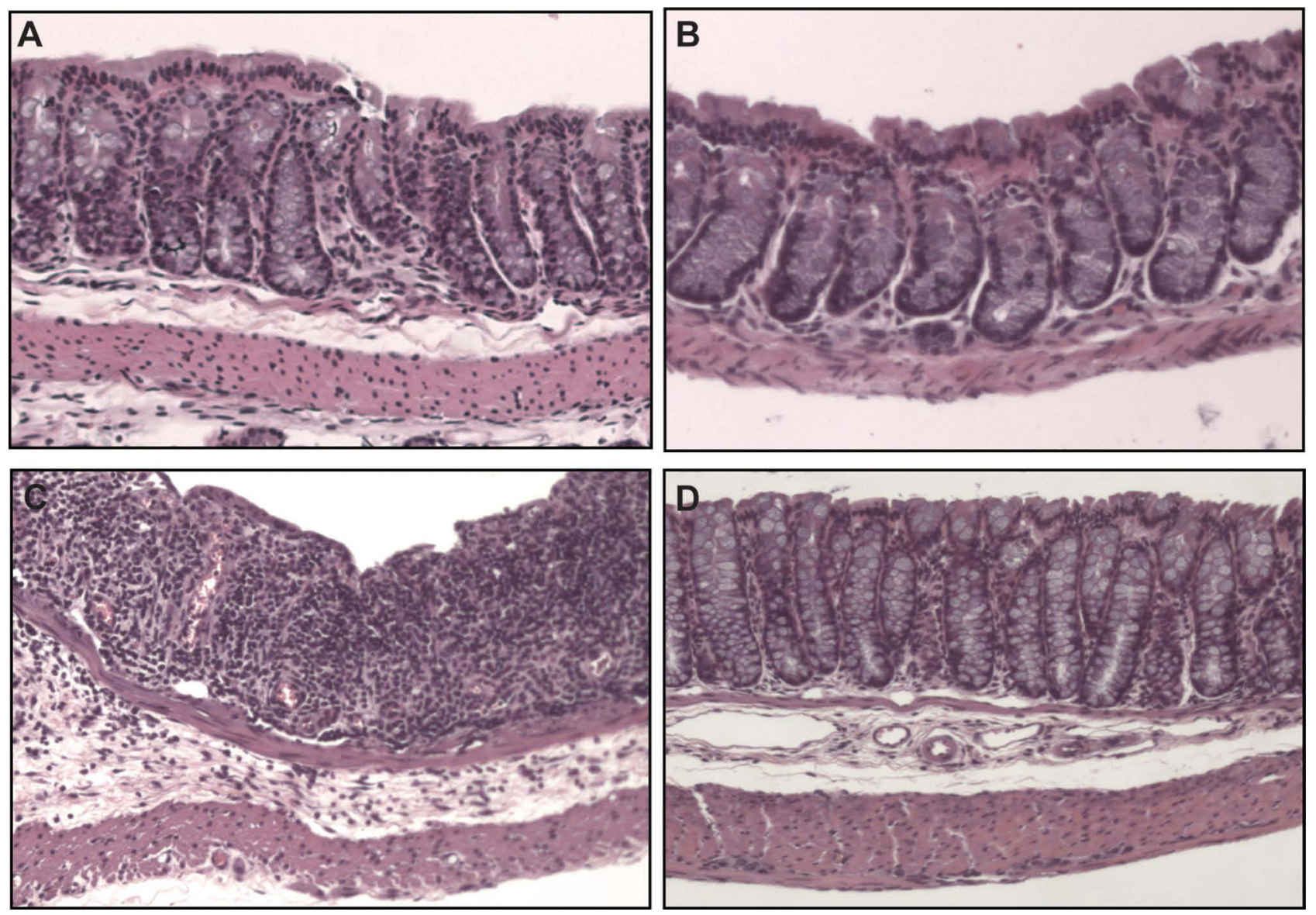

Figure 5 Colon histology (40x magnification). Control animals (A) had similar histologic appearance to those receiving just TXG (B). DSS-only animals (C) had epithelial damage that was extensive and greater than that shown in the DSS+TXG animals (D).

better in DSS treated with TXG $(10.7 \pm 7.9, \mathrm{p}<0.0001)$. The prior literature showing that rectally administered therapies have more consistent efficacy to the left colon was supported by our findings: The TI index was reduced from $26.2 \pm 6.1$ to $15.1 \pm 2.5, \mathrm{p}<0.000$ (Figure 4A). Consistent with left colon TI index scoring, TXG showed the benefit of a profound reduction in crypt abscesses (DSS $2.5 \pm 1.44$, DSS+TXG $0.9 \pm 0.9, \mathrm{p}=0.0075$, Figure 4F), cryptitis (DSS $1.9 \pm 0.6$, DSS+TXG $1.3 \pm$ $0.5, p=0.0203$, Figure 4E), and cellular infiltration (DSS $4.1 \pm 1.0$, DSS + TXG $2.9 \pm 0.6, p=0.0039$, Figure 4B).

\section{Other Morphology}

The addition of TXG was associated with substantial (leftsided) protection against the goblet cell loss caused by DSS (DSS $1.9 \pm 0.6$, DSS+TXG $0.8 \pm 0.5, \mathrm{p}<0.0021$, Figure 4D). Consistent with the reduction in inflammation, TXG administration also demonstrated (Figure 4G, J and $\mathrm{K}$ ) reductions in erosions (DSS $1.8 \pm 0.9$, DSS+TXG $0.9 \pm$ $0.6, p=0.017$, Figure 4G), crypt irregularity (DSS $2.2 \pm$
0.8 , DSS+TXG $0.9 \pm 0.9, \mathrm{p}=0.0026$, Figure $4 \mathrm{~J})$, and crypt loss (DSS $1.7 \pm 1.2$, DSS+TXG $0.6 \pm 0.8, \mathrm{p}=0.026$, Figure $4 \mathrm{~K})$. The measure of ulceration had a trend for protection by TXG compared to DSS alone (DSS $4.0 \pm$ 1.1 , DSS+TXG $3.3 \pm 0.8, p=0.12$, Figure $4 \mathrm{H}$ ). In that there is expected healing of the colon following intermittent dosing of the toxic DSS it was not unexpected that with the addition of TXG there was less epithelial cell hyperplasia (DSS $2.5 \pm 1.2$, DSS+TXG $1.3 \pm 0.8, \mathrm{p}=$ 0.0003 , Figure $4 \mathrm{C}$ ) as well as less granulation tissue (DSS $3.6 \pm 1.4$, DSS+TXG $2.2 \pm 1.3, \mathrm{p}=0.035$, Figure 4I). These improved inflammation parameters for inflammation are consistent with the health-related in vivo measures described above, and support the therapeutic efficacy of two days of intrarectal TXG administration.

\section{Discussion}

The etiologies and optimal treatments have not been fully elucidated for the inflammatory bowel disorders of UC and Crohn's disease. Despite IBD and other causes of colitis 
(such as C. difficile toxin or ischemia) having different primary pathophysiologies, they do share remarkable histologic features of severe inflammation, leukocyte infiltration, loss of colonic macrostructure, denudation of epithelial structures and impaired production and maintenance of the protective mucin layer. Ongoing maintenance and healing of injury to the colon is in great part due to differentiation of "adult" stem cells deep in the crypts. The precursor cells differentiate, proliferate and migrate upwards as they recapitulate first crypt and then villi structures. Migration reportedly involves the motogenic signals from intestinal trefoil factor peptides (eg, TFF3) and interactions with the mucin layer. ${ }^{10}$ As reviewed by Van Klinken et al, ${ }^{11}$ the type of mucin produced changes as the cells regenerate: some beginning with expression of the transmembrane MUC1 and transitioning into the secretory MUC2. ${ }^{12}$ Thus, mature goblet cells mainly produce MUC2 not MUC1, while it is vice versa in colonic absorptive cells. The chemical structure of the various mucins determines their bioadhesion. Based on the literature and our preliminary findings, we believe that a key to understanding the clinical course of colitis is recognition that the integrity of the cellular and mucopolysaccharide milieu is critical for protecting the stem cells and allowing tissue regeneration. Thinning or complete loss of the mucin gel layer on colonic tissue has been well described in surgical specimens of effected bowel from humans with UC. ${ }^{13}$ Hence, it would be no surprise that even with effective treatment of the primary cause of IBD, healing would be secondarily impaired by the loss of the mucin barrier, infiltration by inflammatory cells and cytokines, superinfection by colon bacteria, the risk of abscesses, hemorrhage, perforation, fibrosis, bowel-to-bowel adhesions and obstruction. With a long history of medicinal uses of bioadhesives we advocate further investigation of these naturally derived materials as an adjuvant therapy for IBD: restoration of the chemical and mechanical barrier, resulting in protection of the all-important stem cell niche. Inert biocompatible adhesives can also be derivatized so as to function as drug delivery agents, which would target high concentration local therapy that avoids the toxicity from systemic (eg, intravenous) administration.

To identify an adhesive for the colon we sought a biocompatible polymer similar to the natural mucins. Small and large bowel mucins are large, heterogeneous glycoproteins with molecular weights up to approximately $20 \times$ $10^{6} \mathrm{Da}$, and there is homology across species. ${ }^{14,15}$ The mucins share the motif of tandem repeats of either serine or threonine in the protein core. These serve as the sites for O-linked $\mathrm{N}$ acetyl galactosamine, to which are attached oligosaccharide side chains of diverse sugars and branching. Negative charge (eg, from sulphation of the terminal residues) is thought to be important for tenacity as well as providing resistance to degradation by bacterial enzymes. ${ }^{1}$ Bioadhesion of the various mucins is not fully understood, however. While the sulfate and sialic acid residues may bind to selectins or other adhesion promoting molecules, tenacity may also stem from the underlying filamentous geometric structure that varies across the many mucins. ${ }^{11}$

Plant-based adhesives are very attractive from the biocompatibility standpoint in that many are derived from common foods and have well-established safety in man. Some have already been used for centuries, if not millennia, for a variety of gastrointestinal maladies, salves for skin wounds, glues to close lacerations, and even as eyedrops for corneal abrasions. Studies have been historically challenging in that a single plant or seed can contain a plethora of potential active compounds that are influenced by the cultivation, cooking or purification methodologies. Our interest stems from the chemistry of certain plant nutraceuticals in that their polymeric ultrastructure explains adhesive characteristics. They are amenable to derivatization to both enhance tenacity and confer functionality as local therapeutic agents. ${ }^{16}$ It is important that these plants also have non-adhesive beneficial qualities: derivatives of the extraction processes and solvents could, for example, include soluble glycosides formed in the extraction of the phenol (eg, flavonoids) or terpenoids. These compounds could independently have the confounding effects of altering mucin and TFF3 production, being anti-inflammatory (eg, altering such cytokines as interleukin (IL) 6, 10, and tumor necrosis factor), immunoregulatory (eg, affecting lymphocytes, macrophage activation, interferon), anti-tumor, anti-oxidant (eg, altering nitric oxide production), and changing the microbiome. ${ }^{2,3,17-21}$ Thus, we report extensive analytic testing to verify the structural properties of the tamarind-sourced reagents utilized in this study.

We chose to study XG due to its structure and similarity to mucins. $\mathrm{XG}$ is the polysaccharide predominant in the cell walls of many plants ${ }^{22}$ (ranging from simple grasses to those of higher order) and is highly bound to cellulose. XG confers structural integrity, extensibility, a food reserve and regulation of growth. The polymeric structure has a wide range of up to nearly a micron in length. Instead of the backbone being peptides as in mucins, in $\mathrm{XG}$ it is 
chemically similar to cellulose: a rigid straight chain glucan polymer of up to approximately 3000 glucose molecules in their cyclic (6-carbon glucopyranose) form. Depending on the plant, approximately 30 to $75 \%$ of the glucose residues are attached (asymmetrically to the backbone) to 5-carbon xylose molecules, forming the XG. The xyloses in turn initiate the side chains that are of diverse length, angularity, and made from other sugars (typically the glucose epimer galactose with terminal fucosysl or arabinosyl residues). Digestion of these soluble fibers in man leads to oligosaccharides of various lengths and content. It has been reported that the fragments have the beneficial effect of lowering blood glucose: their galactose content inhibits glucose absorption in a rat intestine model. ${ }^{23}$ There are also reports ${ }^{24}$ of enzymatic degradation of XG by glucanases produced by intestinal bacteria (such as clostridia species), and then gas forming fermentation of the oligosaccharides by other microflora. Biologic effects of oligomeric XG fragments are potentially of high importance but have been poorly studied. ${ }^{25}$ Interpreting the old literature is also challenging in that it is not always clear whether medicinal benefits can be attributed to the polymeric adhesive characteristics, other biologically active qualities of the polymer, or confounded by impurities in the extraction. Independent of the chemical or biologic effects of XG, the water content of the viscous gels is being explored as an approach for drug delivery systems. ${ }^{26,27}$

Rather than contemplating synthesis of $\mathrm{XG}$, there is vast industrial experience in purifying it in large quantities and inexpensively from the seeds of the tamarind fruit (upwards of 80 thousand tons per year for the food industry). Being a hydrogel TXG dissolves in water, and due to its viscosity is then often described as "tamarind gum". ${ }^{28}$ This property makes it an ideal thickening agent commonly used for cooking and as a texturizer for processed foods. ${ }^{6}$ Indeed, tamarind is an ancient remedy in traditional medicine for multiple maladies. It has continued use and study in modern times, ${ }^{29,30}$ especially in alternative medicine for burns, other skin wounds, dry eyes, oral care, corneal damage, dyspepsia, diarrhea and has putative antiviral, anti-inflammatory, anti-oxidant, immunomodulatory, antimutagenic and antitumor effects. Hence, ingestion of the natural seeds of tamarind fruit or their extracts could theoretically have additional therapeutic effects beyond the XG adhesive qualities. For example, extracts of tamarind seeds using certain solvents (eg, ethanol) contained flavonoids, alkaloids, tannin and saponin, and accelerated epidermal wound healing in a mouse model. ${ }^{4}$ In one human study, ${ }^{31}$ a benefit from treating dry eyes with tamarind seed was conversion of the corneal epithelium from a hydrophobic to hydrophilic milieu, thereby facilitating the spread of the tear layer.

To test the therapeutic efficacy of TXG for bowel disorders, we chose the DSS model in mice as it is short, simple, highly reproducible and results in many histopathological features present in human colitis. Perhaps by forming nano-lipocomplexes with luminal medium-chain fatty acids ${ }^{32}$ DSS is thought to be toxic to the intestinal lining. The loss of the barrier to both colon contents and bacteria initiates a cascade of inflammatory processes. As a result, the DSS treated mice develop colitis mimicking human UC, with histology showing erosions, mucosal regeneration with dysplasia, lymphoid follicles, and infiltration of macrophages. This results in shortening of the large intestine and rectal bleeding, which were two measures of TXG efficacy incorporated into this study. Based on reports since the 1990s the optimal range of DSS molecular weight (eg, 36-50 kDa), the dose in drinking water, and the schedule of administration have been established to produce acute, chronic or relapsing inflammation ${ }^{33}$ so as to mimic the remission and relapsing course of UC in man. ${ }^{34}$ Chassaing et $\mathrm{al}^{33}$ have provided the details on DSS administration, which were followed in this study. They also highlight the incomplete understanding of the inflammation hallmarked by lymphocytes (eg, T-helper cells and their cytokine release), multiple interleukins, tumor necrosis and transcription factors, and changes to the microbiota (eg, certain Bacteroides and Clostridium populations increase). It has been proposed that the DSS-induced toxicity includes damage to the goblet cells and resultant decrease in both MUC2 and TFF3. This is supported by MUC2 deficiency in a mouse knockout model being associated with worsened DSSinduced colitis. ${ }^{35}$ They reported ulcerations, loss of colonic architecture, decreased epithelial cell differentiation, and increases in pro-inflammatory cells and cytokines. Use of these animal models is supported by findings in humans wherein there is a decrease in MUC2 (and its precursor) synthesis with increasing severity of UC. ${ }^{36}$ Detrimental effects from the loss of MUC2 is relevant to our study in that we hypothesized that coating the intestinal lining with TXG would protect and thereby facilitate goblet cell regeneration for restoration of the natural mucin barrier. TXG may also be beneficial by binding to free radicals and having antioxidant effects. ${ }^{9}$ MUC1 has 
also been reported to have an important protective role in UC and has been studied in the DSS model. ${ }^{37}$ Conversely, Periasamy et $\mathrm{al}^{38}$ suggest that MUC1 could also have maladaptive harmful consequences due to it being a functional analog of receptors for certain proinflammatory cytokines. They propose that the improved histology from TXG administration is in part due to its docking with MUC1, which would then block the binding of cytokine receptors responsible for the inflammatory cascade (eg, increased IL-1 and IL-6).

In our study, we chose intrarectal administration of TXG so as to minimize potential interactions with DSS, hepatobiliary or pancreatic secretions (eg, digestive enzymes) that could reduce the polymer's efficacy from oral dosing. Those possible factors have not been fully addressed by prior animal studies using solely oral compounds, ${ }^{30,38}$ and is relevant in that some current treatments for human IBD are rectal (eg, steroids). It is well known in man that intrarectal routes of pharmaceutical administration have most of their delivery and benefits in the left colon, ${ }^{8}$ which is important for UC affecting that area of bowel. Our findings are consistent with better leftsided XLG exposure as shown by that scoring data showing more therapeutic efficacy when compared to the right colon, reaching statistical significance for nearly all histologic parameters. This model also allows us to use animal weights as an indirect measure of colitis development as well as overall animal health, and this can be measured throughout the entire experiment. As indicated in Figure $3 \mathrm{~A}$, on D12, intrarectal administration of $\mathrm{TXG}$ resulted in a significant improvement in weight (weight gain), indicating alleviation of colitis. In confirmation of these findings, colon shortening occurring as a result of colitis, was also significantly reversed by intrarectal TXG treatment (Figure 3C). Lastly, intrarectal TXG also decreased the incidence of fecal occult blood (Figure 3B). Altogether, these results support that administration of TXG polymer through the intrarectal route is highly beneficial in improving colitic disease. Notably, this therapeutic efficacy was demonstrated after only two doses (days 2 and 5) in the midst of one week of continued DSS administration, and from the perspective of this being a 12-day model of colitis.

To better understand how intrarectal TXG may be alleviating disease severity, we performed comprehensive quantitative histomorphologic assessment of $H \& E$ stained (as well as descriptive assessments of stem cell immuno-stained) colons from each treatment group. We modified a scoring system developed by Erben et al, which incorporates cellular infiltration, epithelial changes and mucosal architecture. ${ }^{7}$ For the left colon as seen in Figure 4A, intrarectal TXG significantly improved the histology as measured by combining the individual severity scores into the TI index. Both the extent and severity of cellular infiltration were significantly alleviated by intrarectal TXG (Figure 4B). When broken down into specific epithelial features, TXG appeared to specifically improve goblet cell loss (Figure 4D), cryptitis (Figure 4E), crypt abscess formation (Figure 4F), and crypt loss (Figure 4K). On analyzing other aspects of mucosa, TXG was also beneficial in preventing erosions (Figure 4G), and irregularities to the crypt architecture (Figure 4J). Altogether, intrarectal TXG was found to be beneficial in improving multiple histopathological parameters of colitis, in particular, preservation of goblet cell number and crypt architecture as well as a reduction in inflammatory cell infiltrate.

This study has limitations in that the physiology of the TXG protection is not well defined. Future investigations will expand on our hypothesis that $\mathrm{XG}$ protects stem cells and thereby supports the healing regenerative processes by evaluating methods to optimize bioadhesion of $\mathrm{XG}$, including synthesis and use of XG derivatives. ${ }^{39,40}$ Future investigations will also be needed to test whether there are other potential confounding effects of intra-rectal therapies, such as changes to the local microbiome. This mouse model of UC is also limited in the nonhomogeneous left-sided distribution predominance of intra-rectal administration and the time-dependent manifestations of DSS toxicity. For future studies of barrier restoration, it is also important that animal models of IBD have focused on UC, and it is not entirely clear how the pathophysiology and treatments of Crohn's may differ, especially in relation to the focal nature of involvement and heterogeneous mucous thickness. ${ }^{13}$

\section{Conclusion}

In summary, intrarectal $\mathrm{TXG}$ demonstrated substantial benefit to reduce the severity of colitis in the mouse DSS model that resembles human UC. The profound protection for whole animal and histologic parameters supports the further investigation of this plant-based polymer as a mucosal adhesive barrier as primary or adjuvant therapy for human IBD. 


\section{Funding}

This study was supported, in part, by State of Florida Legislative funding.

\section{Disclosure}

The authors report no conflicts of interest in this work.

\section{References}

1. Rhodes JM. Colonic mucus and mucosal glycoproteins: the key to colitis and cancer? Gut. 1989;30(12):1660-1666.

2. Talhouk RS, Karam C, Fostok S, El-Jouni W, Barbour EK. Antiinflammatory bioactivities in plant extracts. $J$ Med Food. 2007;10 (1):1-10. doi:10.1089/jmf.2005.055

3. Mueller M, Hobiger S, Jungbauer A. Anti-inflammatory activity of extracts from fruits, herbs and spices. Food Chem. 2010;122 (4):987-996. doi:10.1016/j.foodchem.2010.03.041

4. Bin Mohamad MY, Akram HB, Bero DN, Rahman MT. Tamarind seed extract enhances epidermal wound healing. Int J Biol. 2011;4(1). doi:10.5539/ijb.v4n1p81

5. Chawananorasest K, Saengtongdee P, Kaemchantuek P. Extraction and characterization of Tamarind (Tamarind indica L.) Seed Polysaccharides (TSP) from three different sources. Molecules. 2016;21(6):775. doi:10.3390/molecules21060775

6. Mishra A, Malhotra AV. Tamarind xyloglucan: a polysaccharide with versatile application potential. J Mater Chem. 2009;19(45):8528. doi:10.1039/b911150f

7. Erben U, Loddenkemper C, Doerfel K, et al. A guide to histomorphological evaluation of intestinal inflammation in mouse models. Int J Clin Exp Pathol. 2014;7(8):4557-U4527.

8. Brown J, Haines S, Wilding IR. Colonic spread of three rectally administered mesalazine (Pentasa) dosage forms in healthy volunteers as assessed by gamma scintigraphy. Aliment Pharmacol Ther. 1997;11(4):685-691. doi:10.1046/j.1365-2036.1997.00193.x

9. Periasamy S, Lin C-H, Nagarajan B, Sankaranarayanan NV, Desai UR, Liu M-Y. Tamarind xyloglucan attenuates dextran sodium sulfate induced ulcerative colitis: role of antioxidation. $J$ Funct Foods. 2018;42:327-338. doi:10.1016/j.jff.2018.01.014

10. Wong WM, Poulsom R, Wright NA. Trefoil peptides. Gut. 1999;44 (6):890-895. doi:10.1136/gut.44.6.890

11. Van Klinken BJ, Dekker J, Buller HA, Einerhand AW. Mucin gene structure and expression: protection vs. adhesion. Am J Physiol. 1995;269(5 Pt 1):G613-627.

12. Chambers JA, Hollingsworth MA, Trezise AE, Harris A. Developmental expression of mucin genes MUC1 and MUC2. J Cell Sci. 1994;107(2):413-424. doi:10.1242/jcs.107.2.413

13. Pullan RD, Thomas GA, Rhodes M, et al. Thickness of adherent mucus gel on colonic mucosa in humans and its relevance to colitis. Gut. 1994;35(3):353-359. doi:10.1136/gut.35.3.353

14. Tytgat K, Buller HA, Opdam FJM, Kim YS, Einerhand AWC, Dekker J. Biosynthesis of human colonic mucin: MUC2 is the prominent secretory mucin. Gastroenterology. 1994;107:1352-1363. doi:10.1016/0016-5085(94)90537-1

15. Tytgat KM, Bovelander FJ, Opdam FJ, Einerhand AW, Buller HA, Dekker J. Biosynthesis of rat MUC2 in colon and its analogy with human MUC2. Biochem J. 1995;309(Pt 1):221-229. doi:10.1042/ bj3090221

16. Arulraj P, Gopal V, Jeyabalan G, Kandasamy CS, Sam Jhonson UC, Venkatanarayanan R. Natural polysaccharides for specific targeting to colon-an assessment. Eur J Biomed Pharm Sci. 2015;2(4):306-328.

17. Hwang D, Jo H, Kim J-K, Lim Y-H. Oxyresveratrol-containing Ramulus mori ethanol extract attenuates acute colitis by suppressing inflammation and increasing mucin secretion. $J$ Funct Foods. 2017;35:146-158. doi:10.1016/j.jff.2017.05.042
18. Lim BO, Yamada K, Nonaka M, Kuramoto Y, Hung P, Sugano M. Dietary fibers modulate indices of intestinal immune function in rats. J Nutr. 1997;127(5):663-667. doi:10.1093/jn/127.5.663

19. Aravind SR, Joseph MM, Varghese S, Balaram P, Sreelekha TT. Antitumor and immunopotentiating activity of polysaccharide PST001 isolated from the seed kernel of Tamarindus indica: an in vivo study in mice. Sci World J. 2012;2012:1-14. doi:10.1100/ 2012/361382

20. Komutarin T, Azadi S, Butterworth L, et al. Extract of the seed coat of Tamarindus indica inhibits nitric oxide production by murine macrophages in vitro and in vivo. Food Chem Toxicol. 2004;42 (4):649-658. doi:10.1016/j.fct.2003.12.001

21. Nie W, Deters AM. Tamarind seed xyloglucans promote proliferation and migration of human skin cells through internalization via stimulation of proproliferative signal transduction pathways. Dermatol Res Pract. 2013;2013:359756. doi:10.1155/2013/359756

22. The FS. Structure and functions of xyloglucan. $J$ Exp Bot. 1989;40 (1):1-11. doi:10.1093/jxb/40.1.1

23. Sone Y, Makino C, Misaki A. Inhibitory effect of oligosaccharides derived from plant xyloglucan on intestinal glucose. J Nutr Sci Vitaminol. 1992;38:391-395. doi:10.3177/jnsv.38.391

24. Hartemink R, Van Laere KMJ, Mertens AKC, Rombouts FM. Fermentation of xyloglucan by intestinal bacteria. Anaerobe. 1996;2 (4):223-230. doi:10.1006/anae.1996.0031

25. Yamanaka S, Mimura M, Urakawa H, Kajiwara K, Shirakawa M, Yamatoya K. Conformation of tamarind seed xyloglucan oligomers. Sen'i Gakkaishi. 1999;55(12):590-596. doi:10.2115/ fiber.55.12_590

26. Newton AM, Indana VL, Kumar J. Chronotherapeutic drug delivery of Tamarind gum, Chitosan and Okra gum controlled release colon targeted directly compressed Propranolol $\mathrm{HCl}$ matrix tablets and in-vitro evaluation. Int $J$ Biol Macromol. 2015;79:290-299. doi:10.1016/j.ijbiomac.2015.03.031

27. Dey S, Nandy BC, De JN, Hasnain MS, Nayak AK. Tamarind gum in drug delivery applications. In: Hasanain MS, Nayak AK, editors. Natural Polysaccharides in Drug Delivery and Biomedical Applications. London, UK: Academic Press; 2019:285-306.

28. Paul SR, Nayak SK, Yogalakshmi Y, et al. Understanding the effect of tamarind gum proportion on the properties of tamarind gum-based hydroethanolic physical hydrogels. Polym Plast Technol Eng. 2018;57(6):450-547. doi:10.1080/03602559.2017.1329435

29. Zanke AA, Ambhore JP, Chavhan SA. A review on tamarind gum and its application. World J Pharm Pharma Sci. 2020;9(5):534-547.

30. Kiniwa R, Miyake M, Kimura SI, Itai S, Kondo H, Iwao Y. Development of muco-adhesive orally disintegrating tablets containing tamarind gum-coated tea powders for oral care. Int J Pharm X. 2019;1:100012.

31. Rolando M, Valente C. Establishing the tolerability and performance of tamarind seed polysaccharide (TSP) in treating dry eye syndrome: results of a clinical study. BMC Ophthalmol. 2007;7:5. doi:10.1186/ 1471-2415-7-5

32. Laroui H, Ingersoll SA, Liu HC, et al. Dextran Sodium Sulfate (DSS) induces colitis in mice by forming nano-lipocomplexes with medium-chain-length fatty acids in the colon. PLoS One. 2012;7(3): e32084. doi:10.1371/journal.pone.0032084

33. Chassaing B, Aitken JD, Malleshappa M, Vijay-Kumar M. Dextran sulfate sodium (DSS)-induced colitis in mice. Curr Protoc Immunol. 2014;104:15-25. doi:10.1002/0471142735.im1525s104

34. Okayasu I, Hatakeyama S, Yamada M, Ohkusa T, Inagaki Y, Nakaya R. A novel method in the induction of reliable experimental acute and chronic ulcerative colitis in mice. Gastroenterology. 1990;98(3):694-702. doi:10.1016/0016-5085(90)90290-H

35. Van der Sluis M, De Koning BA, De Bruijn AC, et al. Muc2-deficient mice spontaneously develop colitis, indicating that MUC2 is critical for colonic protection. Gastroenterology. 2006;131(1):117-129. doi:10.1053/j.gastro.2006.04.020 
36. Tytgat KM, van der Wal JW, Einerhand AW, Buller HA, Dekker J. Quantitative analysis of MUC2 synthesis in ulcerative colitis. Biochem Biophys Res Commun. 1996;224(2):397-405. doi:10.1006/ bbrc.1996.1039

37. Monk JM, Lepp D, Zhang CP, et al. Diets enriched with cranberry beans alter the microbiota and mitigate colitis severity and associated inflammation. $J$ Nutr Biochem. 2016;28:129-139. doi:10.1016/j. jnutbio.2015.10.014

38. Periasamy S, Lin CH, Nagarajan B, Sankaranarayanan NV, Desai UR, Liu MY. Mucoadhesive role of tamarind xyloglucan on inflammation attenuates ulcerative colitis. $J$ Funct Foods. 2018;47:1-10. doi:10.1016/j.jff.2018.05.035
39. Madgulkar AR, Bhalekar MR, Asgaonkar KD, Dikpati AA. Synthesis and characterization of a novel mucoadhesive derivative of xyloglucan. Carbohydr Polym. 2016;135:356-362. doi:10.1016/j. carbpol.2015.08.045

40. Mahajan HS, Tyagi VK, Patil RR, Dusunge SB. Thiolated xyloglucan: synthesis, characterization and evaluation as mucoadhesive in situ gelling agent. Carbohydr Polym. 2013;91(2):618-625. doi:10.1016/j.carbpol.2012.08.077

\section{Publish your work in this journal}

Clinical and Experimental Gastroenterology is an international, peerreviewed, open access, online journal publishing original research, reports, editorials, reviews and commentaries on all aspects of gastroenterology in the clinic and laboratory. This journal is indexed on American Chemical Society's Chemical Abstracts Service (CAS)
The manuscript management system is completely online and includes a very quick and fair peer-review system, which is all easy to use. Visit http://www.dovepress.com/testimonials.php to read real quotes from published authors. 